\title{
Double Orthogonal Projection of Four-Dimensional Objects onto Two Perpendicular Three-Dimensional Spaces
}

\author{
Michal Zamboj ${ }^{1}$ (D)
}

Published online: 16 January 2018

(C) Kim Williams Books, Turin 2018

\begin{abstract}
A method of visualization of four-dimensional objects using a double orthogonal parallel projection onto two orthogonal three-dimensional spaces is proposed. A synthetic approach, with the use of basic descriptive geometry tools of Monge's projection in interactive 3D graphical software, is taken. Descriptive constructions of projections of points, lines, planes and three-dimensional spaces are shown. Methods of measuring true lengths of segments and rotating projections of objects into the modeling space are described. The focus lies on improving the spatial ability and understanding of the four-dimensional space. An example of a construction is carried out on a model of tesseract. All the constructions are analogies of constructions in Monge's projection of a three-dimensional space. With advantages of interactive computer graphics, the constructions are easy to perform, and objects are easy to manipulate with.
\end{abstract}

Keywords Four-dimensional visualization - Multidimensional descriptive geometry $\cdot$ Monge's projection $\cdot$ Tesseract

\section{Introduction}

Understanding of the idea of space and its dimensions has lain in the interest of philosophers, scientists and artists through the ages. Four-dimensional and multidimensional geometry has evolved from being completely absent for the

Electronic supplementary material The online version of this article (doi:https://doi.org/10.1007/ s00004-017-0368-2) contains supplementary material, which is available to authorized users.

Michal Zamboj

zamboj@karlin.mff.cuni.cz

1 Faculty of Mathematics and Physics, Charles University, Sokolovská 83, 18675 Prague 8,

Czech Republic 
ancient world to acceptance in synthetic and analytic geometry in the golden era of geometry in the nineteenth century. General understanding of higher dimensions, based on analogies of the one, two and three-dimensional space, was enhanced by Hinton's essay What is the fourth dimension? in Hinton (1880), his later popularizing textbook (Hinton 1904) and provoked by Abbott's masterpiece novel-Flatland (Abbott 2006). Especially Abbott's book was the author's greatest inspiration for the following text. A brief history of multidimensional geometry and, more importantly, synthetically built four-dimensional geometry can be found in Manning (1956). Though this paper presents a synthetic method of visualization, the analytical point of view of linear geometry is essential for verifications. The analytical approach and differential geometry of four-dimensional space is described in Forsyth (1930). A comprehensive and valuable piece on visualization of points, lines and planes of the four-dimensional space onto planes is in Lindgren and Slaby (1968). Stachel (1990) proves the analogy of right angle theorem in the orthogonal projection of the four-dimensional space onto a plane. The principle of projections onto planes may be easily generalized for higher dimensions. For example, Weiss in (1997) also visualizes $n$-dimensional Euclidean space onto a plane. On the other hand, the more dimensions we want to project, the more projecting planes we need to distinguish the visualized object uniquely. Consequently, more copies of the same point in different projections are obtained. To mentally reconstruct an original image, we would like to have the least number of its projections with as much information as possible. Thus our analogical idea is to project the four-dimensional space onto two three-dimensional spaces. ${ }^{1}$ To recognize the position and visual properties of an object in the four-dimensional space, methods similar to those in three dimensions can be used. Such methods include creating projections after some transformations, projections of shades and shadows or projecting planar sections of the object. These methods would be inapproachable for applications, e.g. visual investigation of four-dimensional curves, without current computer technology. In the last decades, high increase of graphical representations of multidimensional objects with computer graphics using projections (Séquin 2002; Miyazaki 1988; Zachariáš and Velichová 2000), transformations (Kageyama 2016), offset curves (Hoffmann and Zhou 1991; Zhou 1991), lighting (Hanson and Heng 1991; Chilton 1980) and other methods has led into better synthetic intuition and understanding of their properties. These methods may be synthetically derived and used from the basic constructions provided in the current paper, in which only the synthetic construction of a tesseract will be carried out. Four-dimensional solids, such as notoriously known tesseract, are often depicted in central or orthogonal projections onto a plane and have become a part of common mathematical knowledge. On evolution of models of four-dimensional objects (see Volkert 2017). With such an amount of information about the fourth dimension, and 200 years after Gaspard Monge, we will use his method-Monge's projection, described in Monge (1799). Not only do we depict four-dimensional objects, but also, with the possibilities of interactive 3D software (e.g. GeoGebra 5),

\footnotetext{
1 In this sense, for a visualization of a space of $n$-th dimension we need two $(n-1)$-dimensional spaces, which seems to be a dead end, at least for now.
} 
we can manipulate them and study them empirically with no use of analytic representation. Later on, we use the notation 3- or 4-space for three or fourdimensional real space.

Monge's projection consists of a double orthogonal projection of 3-space (with the system of orthogonal axes $x, y, z)$ onto two orthogonal planes- $\pi(x, y), v(x, z)$. One of the planes is chosen to be the drawing plane (a paper, computer screen), and the second plane is rotated around their intersecting line-the $x$ axis, called the reference line, onto the drawing plane such that positive values of the $y$-axis and $z$ axis have opposite orientation (usually $y$ downwards, $z$ upwards). Thus each object has two conjugated images (horizontal and vertical projection), where the conjugated points lie on the line of recall, which is perpendicular to the reference line. Basic constructions in Monge's projection can be found in any textbook on descriptive geometry or technical drawing.

Lately, an analogy of Monge's projection has been well described in Şerbănoiu and Şerbănoiu (2017) for projecting four-dimensional objects onto planes or six three-dimensional spaces. In this paper, we will use the same methods of descriptive geometry in analogy of Monge's projection of a four-dimensional space given by the system of orthogonal axes $x, y, z, w$, but instead of making visualizations in many projections, we will use (in general cases) only projections onto two orthogonal 3-spaces $\Xi(x, y, z), \Omega(x, y, w)$. Again, one of the 3 -spaces is chosen to be the modeling 3-space, and the second is rotated around their intersecting plane ${ }^{2}-$ $\pi(x, y)$, called the reference plane, onto the modeling 3-space such that positive values of the $z$-axis and $w$-axis have opposite orientation (we use $z$ downwards, $w$ upwards). Analogically, each object has two conjugated images, where the conjugated points lie on the line of recall, which is perpendicular to the reference plane. Since we represent our geometric 3-space onto a two-dimensional paper, we must certainly be aware of consequences of such a projection. For simplicity's sake, we assume that we have three-dimensional tools for measuring angles and distances, and we can make three-dimensional transformations in the modeling 3-space. Clearly, we can project our modeling 3-space with any type of parallel or even central projection, both of which give us a realistic picture of three-dimensional projections of the original object, and we can also perform planar constructions to describe its spatial properties. ${ }^{3}$

The structure of the paper is as follows. We start with incidence properties of points, lines, planes and 3-spaces and their special cases of perpendicularity. Metric properties are discussed afterwards. In the end, we use the described constructions to build the final tesseract. Although we tried to keep the structure methodical, we would like to put a different approach forward. For example, it is easier for us to imagine a cube in 3-space than infinite planes in special positions. Therefore, we suggest that the kind reader investigates the tesseract from "A construction of a tesseract" and the attached GeoGebra model (Online Resource 1) at first. It may

\footnotetext{
2 This step has no real visualization for us. Imagine a two dimensional creature trying to rotate something around a line. It is impossible for the creature to do the rotation, but it is still possible to draw the rotated result in its two dimensional plane.

3 In the paper, we use a parallel projection.
} 
also be essential to follow properties of lines, planes and 3-spaces on the tesseract model.

\section{Projecting Points, Lines, Planes and 3-Spaces}

A point $A\left[x_{A}, y_{A}, z_{A}, w_{A}\right]$ in 4-space is represented by two orthogonal projections (Fig. 1a). The point $A_{3}\left[x_{A}, y_{A}, z_{A}, 0\right]$, the $\Xi$-image, is the orthogonal projection of $A$ onto $\Xi(x, y, z)$. The line $\overline{A A_{3}}$, which projects $A$ onto $A_{3}$, is called the $\Xi$-projecting line of the point $A$. The point $A_{4}\left[x_{A}, y_{A}, 0, w_{A}\right]$, the $\Omega$-image, is the orthogonal projection of $A$ onto $\Omega(x, y, w)$ by $\Omega$-projecting line. Furthermore, we denote $A_{1}\left[x_{A}, y_{A}, 0,0\right]$ the $\pi$-image of $A$, which is the orthogonal projection of $A_{3}$ and $A_{4}$ onto the reference plane $\pi(x, y)$, and hence $A_{1}$ is the point of intersection of the line of recall $\overline{A_{3} A_{4}}$ and the plane $\pi(x, y)$ in the modeling 3 -space.

A line $a=\overline{A B}$ is represented by its orthogonal projections $a_{3}=\overline{A_{3} B_{3}}$ onto $\Xi(x, y, z)$ and $a_{4}=\overline{A_{4} B_{4}}$ onto $\Omega(x, y, w)$ (Fig. 1b), which are either lines, or they may become a point and line in special positions. The line $a_{3}$ is the line of intersection of the 3 -space $\Xi(x, y, z)$ and the $\Xi$-projecting plane of the line $a$. The same holds for the line $a_{4}$ as the intersection line of the 3-space $\Omega(x, y, w)$ and the $\Omega$ -projecting plane of the line $a$. The orthogonal projections of the lines $a_{3}$ and $a_{4}$ onto the reference plane $\pi(x, y)$ overlap in the line $a_{1}=\overline{A_{1} B_{1}}$ or a point if the original line $a$ is perpendicular to $\pi(x, y)$. The line $a_{1}$ is the line of intersection of its plane of recall - the plane of lines of recall of points on the given line $a$ (such lines are $\overline{A_{3} A_{4}}$ and $\left.\overline{B_{3} B_{4}}\right)$ and the reference plane $\pi(x, y)$. In the modeling 3 -space, the plane of recall is orthogonal to the reference plane $\pi(x, y)$. The point $Z^{a}$ is the point of intersection of the line $a$ and the 3-space $\Xi(x, y, z)$, and it is called the $\Xi$-trace of the line $a$. The point $W^{a}$ is the point of intersection of the line $a$ and the 3-space $\Omega(x, y, w)$, and it is called the $\Omega$-trace of the line $a$. If $Z^{a} \equiv W^{a}$, the line $a$ intersects the reference plane $\pi(x, y)$, otherwise $a$ and $\pi(x, y)$ are parallel or skew.

Let us describe some special positions of lines with respect to the 3-spaces $\Xi(x, y, z)$ and $\Omega(x, y, w)$ (Fig. 2a). By special position, we mean the position of a line parallel, perpendicular or lying in any of the 3-spaces $\Xi(x, y, z)$ and $\Omega(x, y, w) .{ }^{4}$ Spatial relations in 4-space are well described in Lindgren and Slaby (1968).

A line completely perpendicular to the 3 -space $\Omega(x, y, w)$ is also perpendicular to the reference plane $\pi(x, y)$. Therefore its $\Xi$-image in the 3 -space $\Xi(x, y, z)$ is a line perpendicular to $\pi(x, y)$, while the $\Omega$-image in $\Omega(x, y, w)$ is a point. Its plane of recall is represented by a line in the modeling 3 -space.

A line perpendicular to the reference plane $\pi(x, y)$, but not completely perpendicular to any of the 3-spaces $\Xi(x, y, z)$ and $\Omega(x, y, w)$ is represented by both images overlapping in one line perpendicular to the reference plane $\pi(x, y)$ in the modeling 3-space.

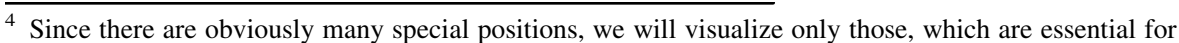
this paper. On the other hand, we would like to encourage the reader to make constructions of her or his own as a valuable exercise.
} 


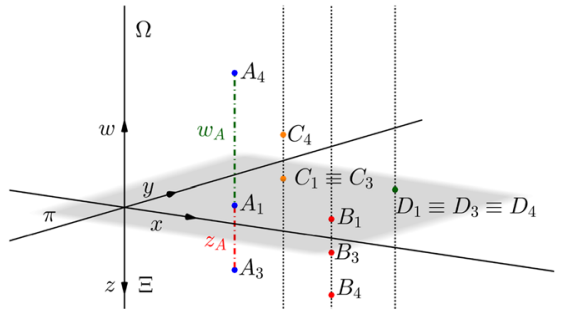

(a)

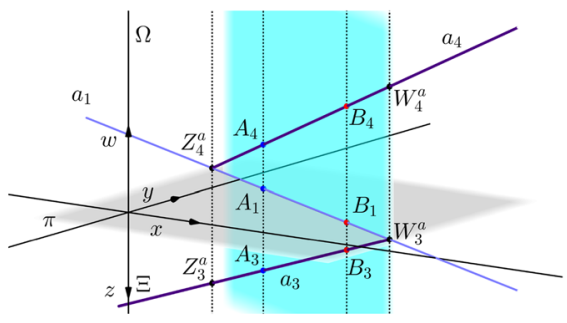

(b)

Fig. 1 a The $\Xi, \Omega$ and $\pi$-images of points $A, B, C$ and $D$. The point $A$ is in a general position with positive $z_{A}$ and $w_{A}$ coordinates. The point $B$ has a negative $w_{B}$ coordinate. The point $C$ has the coordinate $z_{C}=0$. The point $D$ lies in $\pi(x, y)$ and has the coordinates $z_{D}=w_{D}=0$. b A projection of a line $a=\overline{A B}$ in a general position. The $\Xi, \Omega$ and $\pi$-images lie in the plane of recall of the line $a$. The point $Z$ is the $\Xi$ trace and the point $W$ is the $\Omega$-trace of the line $a$

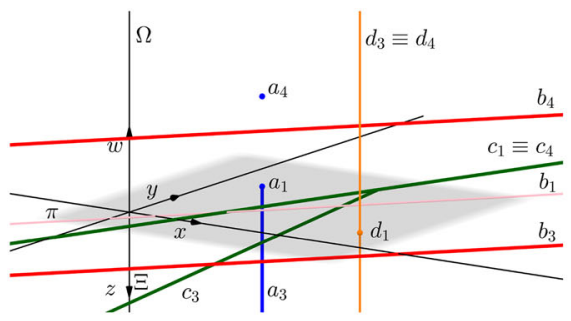

(a)

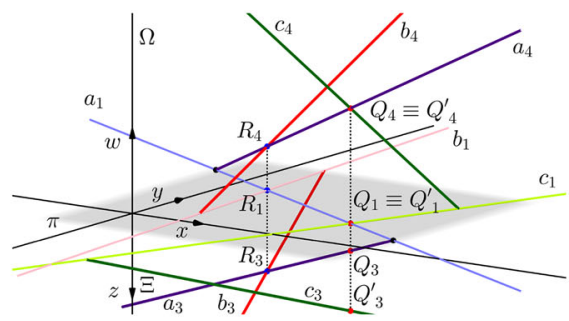

(b)

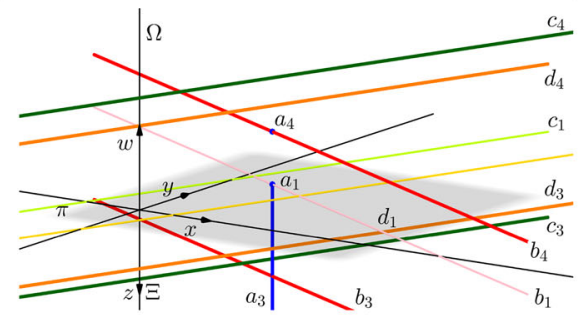

(c)

Fig. 2 a Projection of lines $a, b, c$ and $d$. The line $a$ is perpendicular to the 3 -space $\Omega(x, y, w)$. The $\Xi$ image $a_{3}$ is perpendicular to the reference plane $\pi(x, y)$ and the $\Omega$-image $a_{4}$ becomes a point. The line $b$ is parallel to the reference plane $\pi(x, y)$, and its $\Xi$ and $\Omega$-images are lines parallel to $\pi(x, y)$. The line $c$ lies in the 3-space $\Xi(x, y, z)$, hence its $\Omega$-image $c_{4}$ lies in the reference plane $\pi(x, y)$. The line $d$ is perpendicular to the plane $\pi(x, y)$, but not completely perpendicular to any of the 3 -spaces $\Xi(x, y, z)$ and $\Omega(x, y, w)$, therefore its $\Xi$ and $\Omega$-images overlap in a line perpendicular to the reference plane $\pi(x, y)$. $\mathbf{b}$ Projections of lines $a, b$ and $c$. The lines $a$ and $b$ intersect in a point $R$. Thus the points $R_{3}$ and $R_{4}$ are intersecting points of $a_{3}, b_{3}$ and $a_{4}, b_{4}$, respectively. The lines $a$ and $c$ are skew. The $\Omega$-images of points $Q$ and $Q^{\prime}$ overlap in the point $Q_{4} \equiv Q_{4}^{\prime}$ - the intersection of $a_{4}$ and $c_{4}$, but the points $Q_{3}$ on $a_{3}$ and $Q_{3}^{\prime}$ on $c_{3}$ are distinct. c Projections of lines $a, b, c$ and $d$. The lines $a$ and $b$ are perpendicular, where $a$ is also perpendicular to $\Omega(x, y, w)$. The $\Xi$-images $a_{3}$ and $b_{3}$ are perpendicular to each other in the modeling 3space. The lines $c$ and $d$ are parallel, hence their $\Xi$ and $\Omega$ images are also parallel 
A line parallel to the reference plane $\pi(x, y)$ is represented in both 3-spaces $\Xi(x, y, z)$ and $\Omega(x, y, w)$ by lines parallel to $\pi(x, y)$ in the modeling 3-space.

The $\Omega$-image of a line lying in the 3 -space $\Xi(x, y, z)$ lies in $\pi(x, y)$, while the $\Xi$ image overlaps the original line.

Two distinct lines are intersecting, skew (Fig. 2b) or parallel (Fig. 2c). The $\Xi$ images of two perpendicular lines $a$ and $b$, from which one is parallel to the 3-space $\Xi(x, y, z)$ are perpendicular lines, or one of them is a point. The same holds for the 3 space $\Omega(x, y, w)$. This property of orthogonal projections is called the right-angle theorem, for details in projections onto a plane (see Stachel 1990). Orthogonal projections preserve parallelism, therefore also images of the parallel lines are parallel or they become one line or two distinct points.

A plane $\alpha$ is represented by its conjugated images (Fig. 3a), which are orthogonal projections $\alpha_{3}$ onto $\Xi(x, y, z)$ and $\alpha_{4}$ onto $\Omega(x, y, w)$. The images are either planes, or they are a line and plane or two lines in special positions. The plane $\alpha_{3}$ is the intersection of its $\Xi$-projecting 3-space and the 3-space $\Xi(x, y, z)$. Similarly, the plane $\alpha_{4}$ is orthogonally projected by its $\Omega$-projecting 3 -space onto the 3 -space

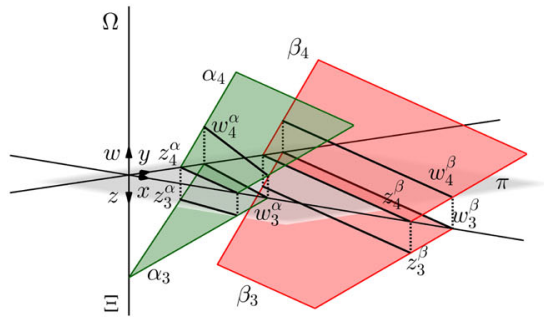

(a)

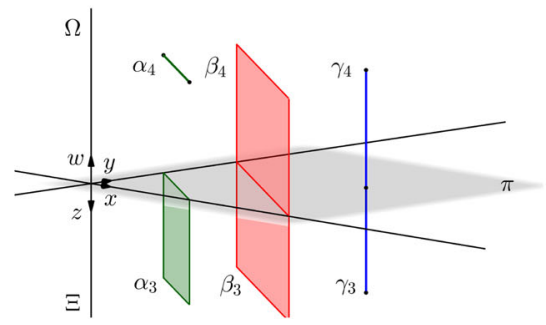

(b)

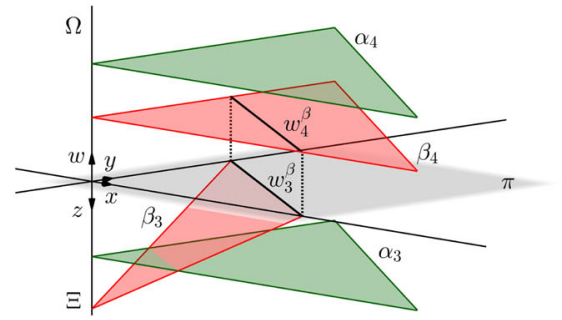

(c)

Fig. 3 a Projections of planes $\alpha$ and $\beta$. The line $z^{\alpha}$ is the $\Xi$-trace and the line $w^{\alpha}$ is the $\Omega$-trace of the plane $\alpha$. The traces are not parallel in the modeling space, hence the plane $\alpha$ intersects the reference plane $\pi(x, y)$ in one point. The plane $\beta$ is parallel to the reference plane $\pi(x, y)$ in one direction. Its traces $w^{\beta}$ and $z^{\beta}$ are parallel lines. b Projections of planes $\alpha, \beta$ and $\gamma$ perpendicular to the reference plane $\pi(x, y)$. The plane $\alpha$ is perpendicular to the 3 -space $\Omega(x, y, w)$ and parallel to the 3 -space $\Xi(x, y, z)$. Its $\Omega$-image $\alpha_{4}$ appears as a line parallel to the reference plane $\pi(x, y)$. The plane $\beta$ is perpendicular to the reference plane $\pi(x, y)$ with a common intersection line. The plane $\gamma$ is perpendicular to the reference plane $\pi(x, y)$ and intersects it in one point. The whole plane appears as the overlapping lines $\gamma_{3} \equiv \gamma_{4}$ in the modeling space. c Projections of planes $\alpha$ and $\beta$ parallel to the 3-space $\Xi(x, y, z)$. The plane $\alpha$ is parallel to the both 3spaces $\Xi(x, y, z)$ and $\Omega(x, y, w)$. Both images $\alpha_{3}$ and $\alpha_{4}$ are parallel to the reference plane $\pi(x, y)$. The plane $\beta$ is parallel to the 3 -space $\Xi(x, y, z)$ but intersects the 3 -space $\Omega(x, y, w)$, and hence only the $\Omega$ image $\beta_{4}$ is parallel to the reference plane $\pi(x, y)$ 
$\Omega(x, y, w)$. The orthogonal projections of the planes $\alpha_{3}$ and $\alpha_{4}$ onto the reference plane $\pi(x, y)$ overlap into the whole reference plane $\alpha_{1} \equiv \pi(x, y)$, or they overlap in a line or point if the original plane $\alpha$ is perpendicular to the reference plane $\pi(x, y)$. In general position, the 3-space of recall of the plane $\alpha$ generated by the lines of recall of its points is the whole modeling space. The trace $z^{\alpha}$, the $\Xi$-trace, is the line of intersection of the 3 -space $\Xi(x, y, z)$ and the plane $\alpha$. The trace $w^{\alpha}$, the $\Omega$-trace, is the line of intersection of the 3-space $\Omega(x, y, w)$ and the plane $\alpha$. If $w_{3}^{\alpha} \equiv z_{4}^{\alpha}$, the plane $\alpha$ and the reference plane $\pi(x, y)$ intersect in a common line, otherwise they intersect in one point, or they are parallel.

Let us discuss some special positions of planes with respect to the modeling space (Fig. 3b, c):

A plane perpendicular to the 3-space $\Omega(x, y, w)$ is also perpendicular to the reference plane $\pi(x, y)$. Therefore its $\Xi$-image in the 3 -space $\Xi(x, y, z)$ is a plane perpendicular to $\pi(x, y)$, while the $\Omega$-image in $\Omega(x, y, w)$ is a line parallel to the reference plane $\pi(x, y)$. Its 3 -space of recall is represented by a plane in the modeling 3-space.

A plane completely perpendicular to the reference plane $\pi(x, y)$, is represented by both images overlapping in one line perpendicular to the reference plane $\pi(x, y)$ in the modeling 3-space. An example of such plane is the plane spanned by the $z$ and $w$ axis through the origin.

The $\Omega$-image of a plane parallel to the 3 -space $\Xi(x, y, z)$ is a plane or line (if it is also perpendicular to $\Omega(x, y, w))$ parallel to the reference plane $\pi(x, y)$.

A plane parallel to the reference plane $\pi(x, y)$ in two distinct directions is represented in both 3 -spaces $\Xi(x, y, z)$ and $\Omega(x, y, w)$ by planes parallel to $\pi(x, y)$ in the modeling 3-space.

The $\Omega$-image of a plane lying in the 3 -space $\Xi(x, y, z)$ lies in the reference plane $\pi(x, y)$, while the $\Xi$-image overlaps the original plane.

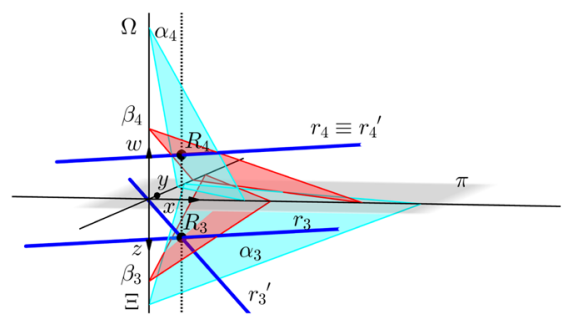

(a)

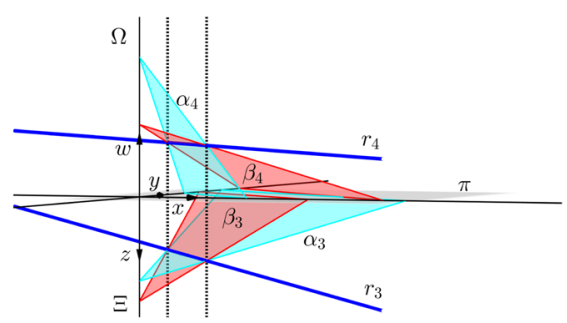

(b)

Fig. 4 a Projections of planes $\alpha$ and $\beta$ intersecting in a point $R$. The intersecting point $R$ lies on a line $r$ in the plane $\beta$ and also on a line $r^{\prime}$ in the plane $\alpha$. The $\Omega$-images of the lines $r$ and $r^{\prime}$ are chosen as the lines overlapping in the line of intersection of $\alpha_{4}$ and $\beta_{4}$, but their $\Xi$-images $r_{3}$ and $r_{3}^{\prime}$ are intersecting lines with the common point $R_{3}$. The $\Omega$-image $R_{4}$ lies on the line $r_{4} \equiv r_{4}^{\prime}$ and on the reference line of the point $R$ through $R_{3}$. b Projections of planes $\alpha$ and $\beta$ intersecting in a line $r$. The images $r_{3}$ and $r_{4}$ of the line of intersection of the planes $\alpha$ and $\beta$ are the lines of intersection of the images $\alpha_{3}, \beta_{3}$ and $\alpha_{4}, \beta_{4}$ of the planes $\alpha$ and $\beta$ 


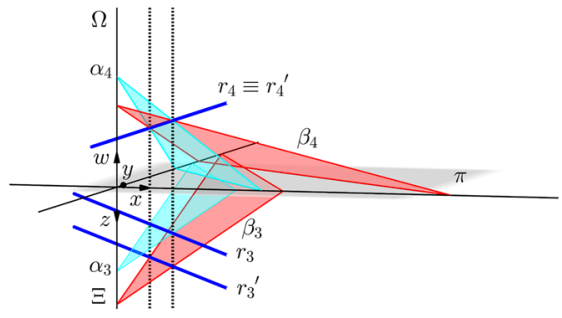

(a)

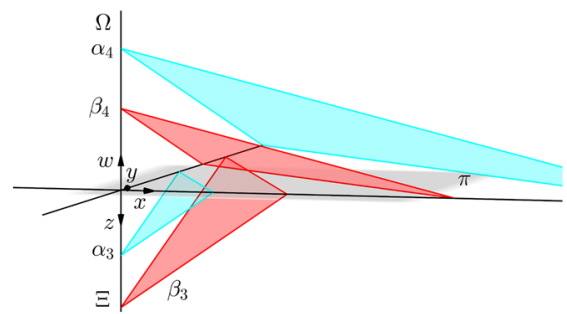

(b)

Fig. 5 a Projections of planes $\alpha$ and $\beta$ parallel in one direction. The $\Omega$-images $\alpha_{4}$ and $\beta_{4}$ intersect in the overlapping $\Omega$-images $r_{4} \equiv r_{4}^{\prime}$ of the lines $r$ and $r^{\prime}$. The $\Xi$-images $r_{3}$ in $\alpha_{3}$ and $r_{3}^{\prime}$ in $\beta_{3}$ are parallel lines. b Projections of planes $\alpha$ and $\beta$ parallel in two directions. The $\Xi$-images $\alpha_{3}, \beta_{3}$ and also the $\Omega$-images $\alpha_{4}, \beta_{4}$ of the planes are parallel

Two distinct planes in 4-space intersect in a point (Fig. 4a), in a line (Fig. 4b), or they are parallel (Fig. 5a, b). To determine the position of two planes $\alpha$ and $\beta$ we find the intersections of their $\Xi$-images and $\Omega$-images. Let $r_{4}$ be the intersection line of $\alpha_{4}$ and $\beta_{4}$. The intersections of the plane of recall of the line $r_{4}$ with the $\Xi$-images $\alpha_{3}$ and $\beta_{3}$ are two distinct intersecting or parallel lines $r_{3}$ and $r_{3}^{\prime}$ (hence $r_{4} \equiv r_{4}^{\prime}$ is overlapping $\Omega$-image of lines $r$ and $r^{\prime}$ ) or coincident lines. If $r_{3}$ and $r_{3}^{\prime}$ are intersecting lines, the planes $\alpha$ and $\beta$ intersect in one point $R$, where $R_{3}$ is the point of intersection of $r_{3}$ and $r_{3}^{\prime}$ and $R_{4}$ lies on the line $r_{4} \equiv r_{4}^{\prime}$ and its line of recall through $R_{3}$. If $r_{3}$ and $r_{3}^{\prime}$ are parallel, the planes $\alpha$ and $\beta$ are parallel (they have no intersection and one common direction). If $r_{3} \equiv r_{3}^{\prime}$, the planes $\alpha$ and $\beta$ intersect in the line $r$. Finally, if $\alpha_{4}$ and $\beta_{4}$ and also $\alpha_{3}$ and $\beta_{3}$ do not intersect, the planes $\alpha$ and $\beta$ are parallel.

The following property of an orthogonal projection is useful for constructions with special planes and lines of 3 -spaces (Fig. $6 \mathrm{a}-\mathrm{c}$ ). The $\Xi$-images of a line $k$ perpendicular to a plane $\alpha$, from which one is parallel to the 3-space $\Xi(x, y, z)$, are also perpendicular, or the image $k_{3}$ of the line is a point, or the image $\alpha_{3}$ of the plane is a line perpendicular to the image $k_{3}$. The same holds for the $\Omega$-images and the space $\Omega(x, y, w)$.

The orthogonal projection of a 3-space $\Sigma$ onto a non-orthogonal 3-space is the whole 3 -space. The orthogonal projection of a 3 -space $\Sigma$ onto an orthogonal 3-space is a plane. We represent a 3 -space $\Sigma$ by its intersections with $\Xi(x, y, z)$ and $\Omega(x, y, w)$ (Fig. 7a). The trace $\xi^{\Sigma}$, the $\Xi$-trace, is the plane of intersection of the 3space $\Xi(x, y, z)$ and the 3 -space $\Sigma$. The trace $\omega^{\Sigma}$, the $\Omega$-trace, is the plane of intersection of the 3 -space $\Omega(x, y, w)$ and the 3 -space $\Sigma$. If the 3 -space $\Sigma$ is not parallel to $\Xi(x, y, z)$ or $\Omega(x, y, w)$, then it intersects $\pi(x, y)$ in a line, which is common for both traces of $\Sigma$. Planes of the 3 -space $\Sigma$, which are parallel to the $\Xi(x, y, z)$ or $\Omega(x, y, w)$ are called $\Xi$-planes or $\Omega$-planes of $\Sigma$, respectively. Lines of the 3 -space $\Sigma$, perpendicular to its $\Xi$-plane or $\Omega$-plane, are called the $\Xi$-slope line or $\Omega$-slope line, respectively.

Let us have one image, for instance $A_{4}$, of a point $A$, lying in a 3 -space $\Sigma$ given by its traces. We can conveniently use the $\Xi$ or $\Omega$-planes through the point $A$ to 


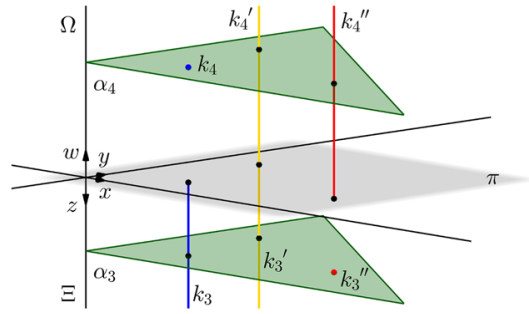

(a)

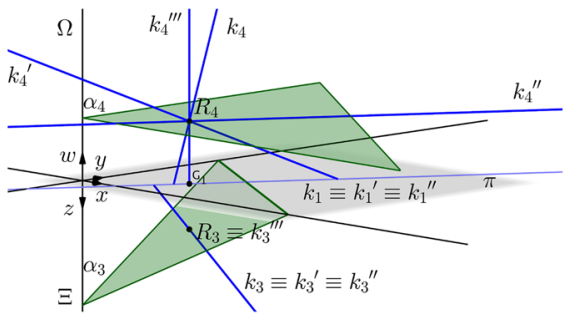

(b)

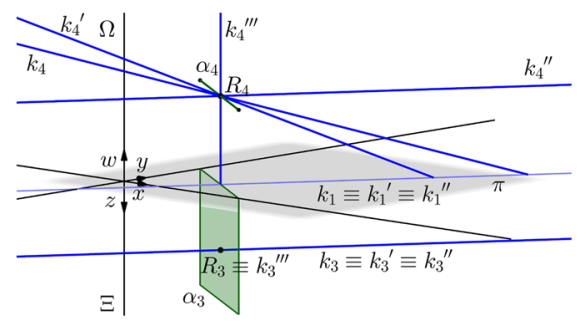

(c)

Fig. 6 Projections of all the different positions of lines $k, k^{\prime}, k^{\prime \prime}$ and $k^{\prime \prime \prime}$ perpendicular to a plane $\alpha$. The plane $\alpha$ is in all three cases parallel to the 3-space $\Xi(x, y, z)$. On the other hand, the figure also interprets positions of the plane $\alpha$ perpendicular to different types of lines parallel to the 3 -space $\Omega(x, y, w)$, which are described below. a The plane $\alpha$ is also parallel to the 3 -space $\Omega(x, y, w)$. The line $k^{\prime \prime}$ is parallel to $\Omega(x, y, w)$. b The plane $\alpha$ intersects the 3-space $\Omega(x, y, w)$ in a line. The line $k^{\prime \prime \prime}$ is parallel to $\Omega(x, y, w)$. c The plane $\alpha$ is perpendicular to the 3-space $\Omega(x, y, w)$. All the lines $k, k^{\prime}, k^{\prime \prime}$ and $k^{\prime \prime \prime}$ are parallel to $\Omega(x, y, w)$

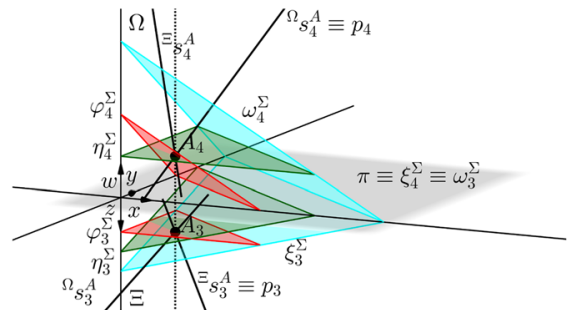

(a)

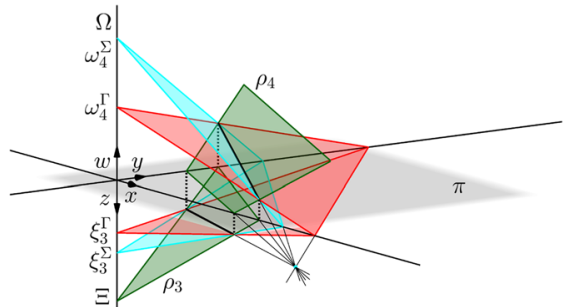

(b)

Fig. 7 a Projections of a 3 -space $\Sigma$ represented by its traces $\xi^{\Sigma}$ and $\omega^{\Sigma}$. The plane $\eta$ is the $\Xi$-plane and the plane $\varphi$ is the $\Omega$-plane of the 3-space $\Sigma$. The point $A$ lies in the 3 -space $\Sigma$ and also in the planes $\eta$ and $\varphi$. The lines ${ }^{\Xi} s^{A}$ and ${ }^{\Omega} s^{A}$ are the $\Xi$ and $\Omega$-slope lines of the 3 -space $\Sigma$ through the point $A$. The line $p$ through the point $A$ is perpendicular to the 3 -space. $\Sigma \mathbf{b}$ Projections of 3 -spaces $\Sigma$ and $\Gamma$ intersecting in a plane $\rho$. The intersecting lines of the 3 -spaces $\Sigma$ and $\Gamma$ with the reference plane $\pi(x, y)$ and also the $\Xi$ and $\Omega$-traces of $\rho$ meet in one point of the modeling space

determine the second conjugated image. Let $\eta_{4}^{\Sigma}$ be the $\Xi$-plane of $\Sigma$ through the point $A$. The intersection of $\eta_{4}^{\Sigma}$ and the $\Omega$-trace $\omega_{4}^{\Sigma}$ is the $\Omega$-image of the $\Omega$-trace of $\eta^{\Sigma}$. The $\Xi$-image of the $\Omega$-trace of $\eta^{\Sigma}$ is the intersection of its plane of recall and the reference plane $\pi(x, y)$. The $\Xi$-image $\eta_{3}^{\Sigma}$ of the plane $\eta^{\Sigma}$ passes through the $\Xi$-image of its $\Omega$-trace and is parallel to the $\Xi$-trace of the 3 -space $\Sigma$. 
Two distinct 3-spaces are parallel, if their $\Xi$-traces and $\Omega$-traces are parallel. Otherwise, at least one pair of traces are intersecting planes, and the 3-spaces intersect in a plane (Fig. 7b). Let us find the plane of intersection $\rho$ of two 3-spaces $\Sigma$ and $\Gamma$ given by their traces. At first, we find the intersecting line of one pair of the traces, for example $\omega_{4}^{\Sigma}$ and $\omega_{4}^{\Gamma}$. This line is the $\Omega$-image of the $\Omega$-trace of the plane $\rho$, and its $\Xi$-image is in the reference plane $\pi(x, y)$. If the $\Xi$-traces $\xi_{3}^{\Sigma}$ and $\xi_{3}^{\Gamma}$ are parallel, then the $\Xi$-image $\rho_{3}$ is the parallel plane through the $\Xi$-image of the $\Omega$ trace of the plane $\rho$. In case of $\xi_{3}^{\Sigma}$ and $\xi_{3}^{\Gamma}$ are intersecting planes, the join of the line of their intersection, the $\Xi$-image of the $\Xi$-trace of the plane $\rho$, with the $\Xi$-image of the $\Omega$-trace of the plane $\rho$, is the $\Xi$-image $\rho_{3}$. The existence of the plane $\rho_{3}$ can be concluded from the following fact: Since the $\Omega$ and $\Xi$-traces of the plane $\rho$ lie in the $\Xi$ and $\Omega$-traces of both 3 -spaces $\Sigma$ and $\Gamma$, they all intersect in one point on the reference plane $\pi(x, y)$, or the $\Omega$ and $\Xi$-traces of the plane $\rho$ are parallel. Therefore, the $\Xi$-image of the $\Omega$-trace and the $\Xi$-image of the $\Xi$-trace of the plane $\rho$ generate the plane $\rho_{3}$.

The $\Xi$ and $\Omega$-images of a line $p$ perpendicular to a 3 -space $\Sigma$ are perpendicular also to its traces $\xi^{\Sigma}$ and $\omega^{\Sigma}$. From the discussion on perpendicularity of lines and planes in special positions, we conclude that $p_{3}$ is perpendicular to $\xi_{3}^{\Sigma}$ and $p_{4}$ is perpendicular to $\omega_{4}^{\Sigma}$.

\section{Measuring Lengths and Angles}

Elementary constructions for measuring lengths and angles in four dimensions will be described in this section. If an object lies in the 3-space $\Xi(x, y, z)$ or $\Omega(x, y, w)$ or in the parallel 3-space to either one of them, then its true shape appears in the modeling 3-space. Otherwise, we need to rotate projecting planes of lines, projecting 3-spaces of planes, or to rotate the whole 3-space around its trace plane, onto the modeling 3-space. After such a rotation we obtain any three-dimensional object of any 3 -space in its true size in the modeling 3-space. ${ }^{5}$

Let us have a segment $A B$ given by its two conjugated images $A_{3} B_{3}$ and $A_{4} B_{4}$ (Fig. 8). To measure the length of the segment we rotate the $\Xi$-projecting plane of the line $a=\overline{A B}$ (or the right-angle trapezoid $A_{3} B_{3} B A$ ) onto the modeling 3-space. We choose an arbitrary plane containing the $\Xi$-image $a_{3}$ and draw the perpendicular lines $a^{\prime}$ and $b^{\prime}$ in this plane to $a_{3}$ through $A_{3}$ and $B_{3}$, respectively. The length of the segment $A_{3} A$ is the $w$-coordinate of the point $A$, or simply the distance between $A_{4}$ and $\pi(x, y)$. The last step is to construct the right-angle trapezoid $A_{3} B_{3}(A)(B)$, where the points $(A),(B)$ lie on $a^{\prime}, b^{\prime}$ and $\left|A_{3}(A)\right|=w_{A},\left|B_{3}(B)\right|=w_{B}$. Another remark of this construction is the visualization of the true angle between the line $a$ and the 3space $\Xi(x, y, z)$, since it is also the angle $\varangle\left(a, a^{\prime}\right)=\varangle\left(a^{\prime},(a)\right)$, where $(a)=\overline{(A)(B)}$. We can conveniently use the $\Xi$-trace of the line $a$ with zero $w$-coordinate.

\footnotetext{
5 Even if we cannot visualize the process of rotation of a 3-space around a plane in 4-space, we can create the resulting model in the modeling 3 -space.
} 


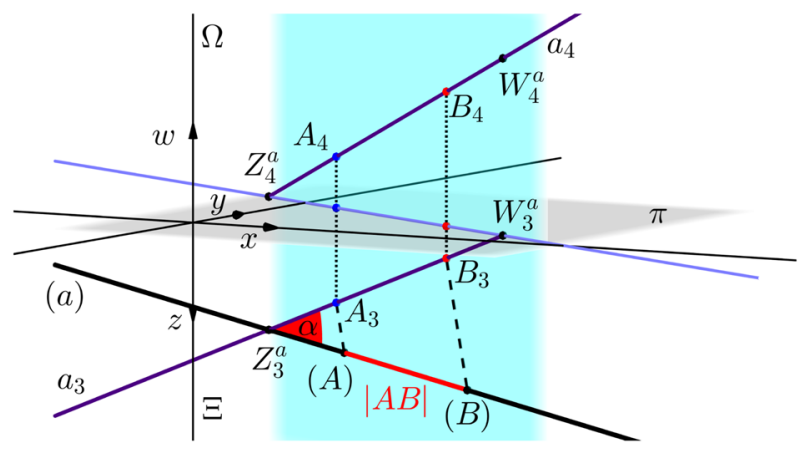

Fig. 8 The construction of the true length $|A B|$ of a segment $A B$ and the true angle $\alpha$ between a line $a$ and the 3 -space $\Xi(x, y, z)$. As an arbitrary plane for the rotation, the projecting plane of the line $a$ is chosen

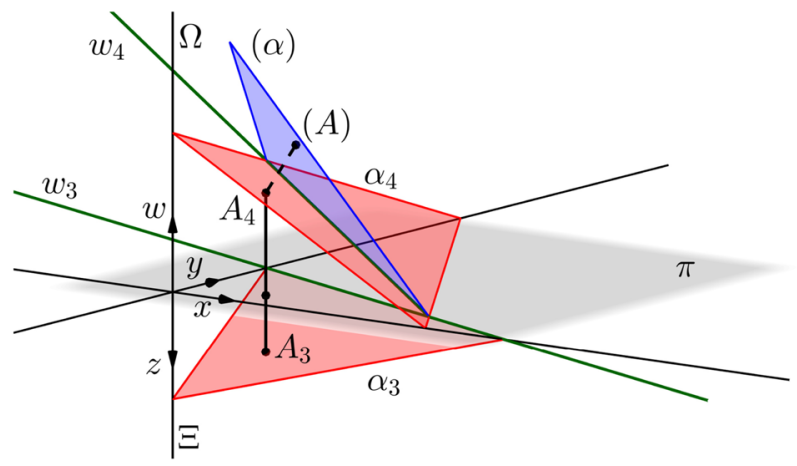

Fig. 9 A rotation of the $\Omega$-projecting 3-space of a plane $\alpha$

The true size of a planar object appears after a rotation of the projecting space of its plane. Let us have a plane $\alpha$ given by its conjugated images and a point $A$ in $\alpha$ (Fig. 9). We rotate the $\Omega$-image $\alpha_{4}$ onto $(\alpha)$. The point $(A)$ lies on the perpendicular line to the $\Omega$-image $\alpha_{4}$ through the point $A_{4}$. The length of the segment $A_{4}(A)$ is equal to $z_{A}$. The $\Omega$-trace $w$ of the plane $\alpha$ is the fixed line of the rotation. The plane $(\alpha)$ is generated by the point $(A)$ and the line $w_{4}$. There is an axial affinity between two planes $\alpha_{4} \rightarrow(\alpha)$ in the modeling 3-space. The axis of the affinity is $w_{4}$ and the direction of the affinity is perpendicular to $\alpha_{4}$. The affinity can be easily used for rotated images of other points, if needed. ${ }^{6}$

Let us have a 3 -space $\Sigma$ in a general position with respect to $\Xi(x, y, z)$ and $\Omega(x, y, w)$ and a point $A$ in $\Sigma$ (Fig. 10). A three-dimensional object in $\Sigma$ is orthogonally projected onto the modeling 3-space. To visualize the object in its true shape we rotate the 3 -space $\Sigma$ around its $\Omega$-trace $\omega^{\Sigma}$ onto the modeling 3-space. The plane $\omega^{\Sigma}$ is the plane of fixed points of the rotation. Let $W^{s}$ be the point of

\footnotetext{
${ }^{6}$ In our two-dimensional figures, there is also a planar affinity that maps the plane $\alpha$ to $(\alpha)$. The axis is the same as in the spatial affinity between two planes and the direction is the projected spatial direction onto our drawing plane.
} 


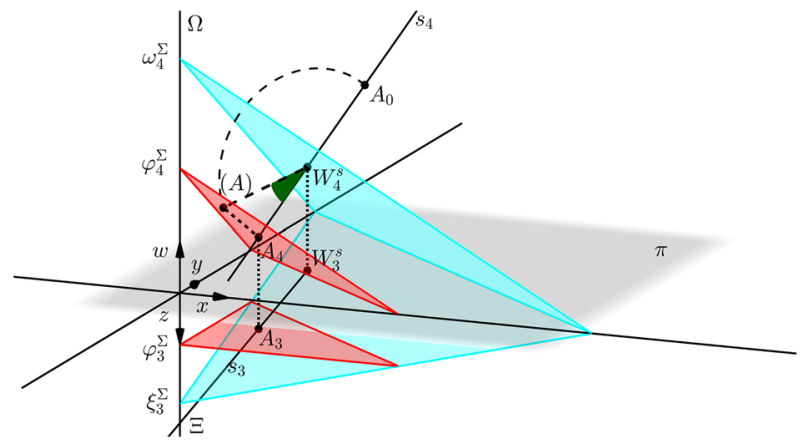

Fig. 10 A rotation of a 3 -space $\Sigma$ onto $\Omega(x, y, w)$

intersection of the $\omega^{\Sigma}$ and the $\Omega$-slope line $s$ through $A$. The triangle $A A_{4} W^{s}$ with a right angle in $A_{4}$ can be constructed in the true size by rotating the $\Omega$-projecting plane of $s$, as above. Afterwards, the segment $W_{4}^{s}(A)$ is rotated to $W_{4}^{s} A_{0}$, where $A_{0}$ lies on the line $s_{4}=\overline{W_{4}^{s} A_{4}}$. The image of any other point of $\Sigma$ can be constructed using the affinity between $\Sigma_{4}$ and $\Sigma_{0}$ in the modeling 3 -space, with the axial plane $\omega_{4}^{\Sigma}$ and the direction of the $\Omega$-slope line $s_{4}$. The same process can be applied for the rotation around the $\Xi$-trace of $\Sigma$.

\section{Projecting Solids}

To investigate a four-dimensional space we use analogical methods to those in a three-dimensional space. We show the double orthogonal projection of a tesseract onto the 3-spaces $\Xi(x, y, z)$ and $\Omega(x, y, w)$. With the use of interactive software, ${ }^{7}$ properties of the projection with respect to the modeling 3-space can be analyzed.

\section{A Construction of a Tesseract}

Let us construct a tesseract $A B C D E F G H I J K L M N O P$ with the cube $A B C D I J K L$ in a 3 -space $\Sigma$, given by its $\Xi$ and $\Omega$-traces. Moreover, let the face $A B C D$ lie in the $\Omega$ trace $\omega^{\Sigma}$, and let the side $A B$ lie on a line parallel to $\pi(x, y)$ (Fig. 11, Online Resource 1).

Since the face $A B C D$ lies in $\omega^{\Sigma}$, it lies also in the 3-space $\Omega(x, y, z)$ and its $\Omega$ image $A_{4} B_{4} C_{4} D_{4}$ is a square with the side $a=|A B|=\left|A_{4} B_{4}\right|$ in its true shape in the modeling 3 -space. For the same reason, the $\Xi$-projection $A_{3} B_{3} C_{3} D_{3} \equiv A_{1} B_{1} C_{1} D_{1}$ lies in the reference plane $\pi(x, y)$.

To construct the cell $A B C D E F G H$ we construct the point $E$ which lies on the perpendicular line $p$ to the 3 -space $\Sigma$ through $A$. The $\Xi$-image $p_{3}$ is perpendicular to $\xi_{3}^{\Sigma}$ and $\Omega$-image $p_{4}$ is perpendicular to $\omega_{4}^{\Sigma}$ in the modeling space. The length of the

\footnotetext{
${ }^{7}$ Step-by-step construction on an interactive model in GeoGebra5 is in the supplementary files: file01_tesseract.ggb.
} 


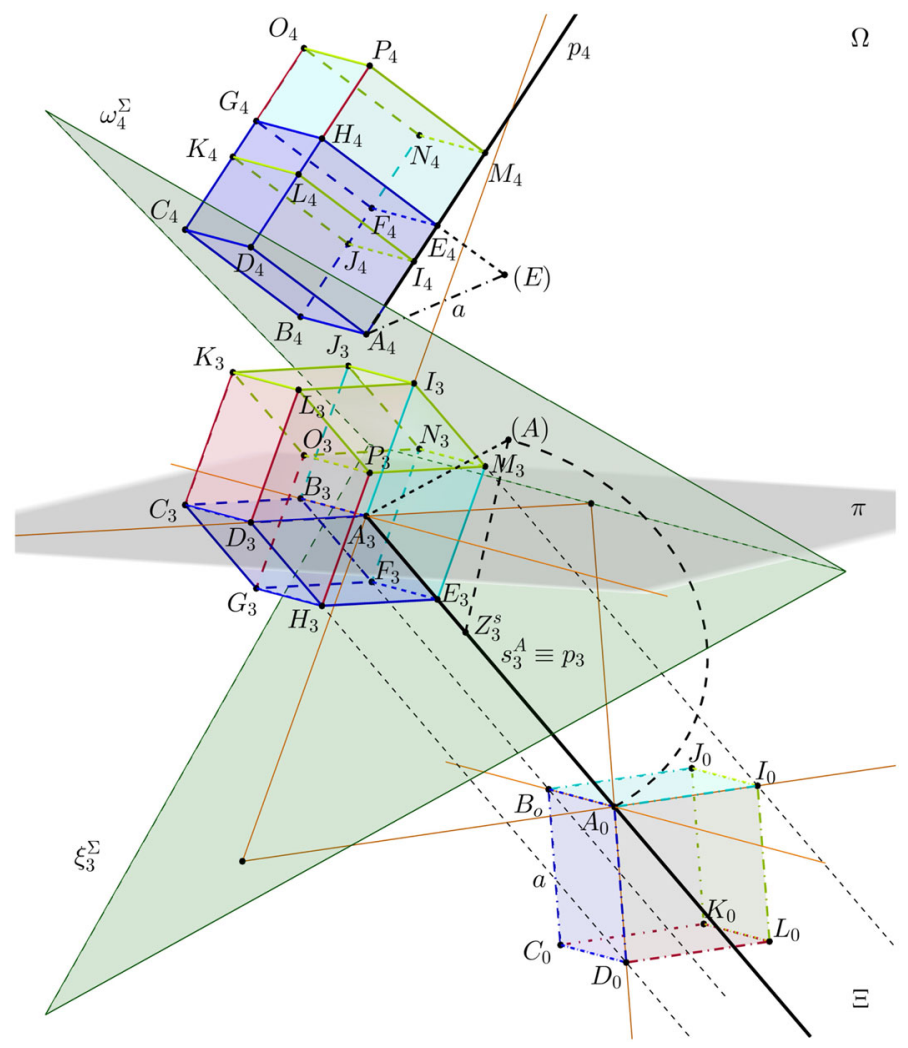

Fig. 11 A construction of a tesseract

edge $A E$ in its true size is $|A E|=a$. Following the construction in Fig. 8, we rotate the $\Omega$-projecting plane of the line $p$ onto the 3 -space $\Omega(x, y, w)$ and find $(p)$. The $\Omega$ trace of the line $p$ is the point $A$, and hence $A_{4} \equiv(A)$. To find the second point of $(p)$, we construct the $\Xi$-trace $Z^{p}$ and its image $\left(Z^{p}\right)$ in the rotation (not depicted in Fig. 11). The point $(E)$ lies on $(p)$ and $\left|A_{4}(E)\right|=a$. The orthogonal projection of $(E)$ onto $p_{4}$ is the point $E_{4}$. The face $E F G H$ is parallel to the face $A B C D$. The $\Omega$-image $E_{4} F_{4} G_{4} H_{4}$ is a square parallel to $A_{4} B_{4} C_{4} D_{4}$. The $\Xi$-image $E_{3}$ of the vertex $E$ is the intersection of the line of recall through the point $E_{4}$ with $p_{3}$. The $\Xi$-image $E_{3} F_{3} G_{3} H_{3}$ is a parallelogram parallel to $A_{3} B_{3} C_{3} D_{3}$.

The next step is to construct the cell $A B C D I J K L$ in the 3 -space $\Sigma$. To do so, we rotate the 3 -space $\Sigma$ around its trace $\xi^{\Sigma}$ onto the 3 -space $\Xi(x, y, z)$. In the rotated 3space $\Sigma_{0}$ we construct the cube $A B C D I J K L$, which appears in its true shape as $A_{0} B_{0} C_{0} D_{0} I_{0} J_{0} K_{0} L_{0}$. Following the construction in Fig. 10, the point $A_{0}$ lies on the $\Xi$-image of the slope line $s_{3}^{A}$ with the $\Xi$-trace $Z^{s}$. The distance $\left|Z_{3}^{s} A_{0}\right|$ equals the distance $\left|Z^{s} A\right|$, which is constructed as the true length $\left|Z_{3}^{s}(A)\right|$ in the right-angle triangle $A_{3}(A) Z_{3}^{s}$ by a rotation of the $\Xi$-projecting plane of the line $s^{A}$ onto $\Xi(x, y, z)$. To complete the $\Xi$-image of the cube $A B C D I J K L$, it is enough to find the point $I_{0}$ 
and its original point $I_{3}$. For a better demonstration, the rotated image of the whole cube is constructed. The points $B_{0}, C_{0}$ and $D_{0}$ lie on their $\Xi$-slope lines through $B_{3}, C_{3}$ and $D_{3}$, respectively. In the figure, the edge $A B$ is parallel to $\xi^{\Sigma}$. Thus also $A_{0} B_{0}$ is parallel to $A_{3} B_{3}$. The point $D_{0}$ is constructed in the affinity between the $\Xi$ image of $\Sigma_{3}$ and the rotated image $\Sigma_{0}$. The fixed point of the line $A_{3} D_{3}$ is its intersection with $\xi_{3}^{\Sigma}$. The join of the fixed point with $A_{0}$ is the rotated image of the line $A_{3} D_{3}$ and its intersection with the $\Xi$-slope line through $D_{3}$ is the point $D_{0}$. With the use of parallelism, distances or affinity we can complete the face $A_{0} B_{0} C_{0} D_{0}$ and also the face $I_{0} J_{0} K_{0} L_{0}$. In the affinity, we find the point $I_{3}$ in the direction of the slope lines and on the original line of the line $A_{0} I_{0}$. The $\Xi$-image of the face $I_{3} J_{3} K_{3} L_{3}$ is parallel to the face $A_{3} B_{3} C_{3} D_{3}$. Since the edge $A I$ is perpendicular to the face $A B C D$ and lies in the 3 -space $\Sigma$, the $\Omega$-image $I_{4}$ lies on the line $p_{4}$ and on the line of recall through $I_{3}$. The remaining points $J_{4}, K_{4}$ and $L_{4}$ form the square parallel to $A_{4} B_{4} C_{4} D_{4}$,

In the last step, we construct the cube IJKLMNOP parallel to the cube $A B C D E F G H$. The vertex $M$ lies on the edge $I M$ parallel to $A E$ and $\left|I_{3} M_{3}\right|=\left|A_{3} E_{3}\right|$ and $\left|I_{4} M_{4}\right|=\left|A_{4} E_{4}\right|$. The face $M N O P$ is parallel to the face $I J K L$, and so are their $\Xi$ and $\Omega$-images.

\section{Conclusion}

Monge's orthographic projection in three-dimensional geometry is not only a tool for constructions in technical drawing, it is also a method of visualizing the threedimensional space in the drawing plane and studying its properties. We have used the same concept of two orthogonal projections to study and visualize a fourdimensional space in a three-dimensional modeling 3-space. It is true that we, threedimensional people, will probably never naturally see the four-dimensional objects as a whole. But none of us has seen a point, a line, a plane or a 3-space either. The more important question raises: Can we visualize it in our mind, can we imagine it? There were many successful attempts to visualize the objects and their dynamic transformations, mostly by computer graphics. There are also methods of constructing four-dimensional objects in projections onto planes. We tried to add a brick into this wall, not only by showing the objects in projections onto two 3-spaces, but also building them constructively and independently of their analytic representations, using simple descriptive geometry tools. With the power of interactive graphics, we are able to move the objects and discover their properties intuitively, enhancing our spatial ability in four and multi-dimensional spaces. An example of such investigation was given on the synthetic construction of the graphical model of a tesseract. We must confess that there was nothing more than curiosity and joy at the beginning of our journey, but the simplicity of the given method leads us to future concerns such as constructions of sections and shadows of four-dimensional solids, synthetic classification of three-dimensional quadrics as section cuts of a sphere cone, creating synthetic models of the projective extension 
of the three-dimensional real space, constructing ruled hypersurfaces in 4-space, and others.

Acknowledgement The work was supported by the grant SVV2017 No. 260454. I also wish to thank Lukas Krump (Charles University) for valuable comments on preliminary versions of the text.

\section{References}

Abbott, Edwin Abbott. 2006. Flatland, a Romance of Many Dimensions. Edited with an Introduction and Notes by Rosemary Jann, Oxford University Press.

Chilton, Bruce L. 1980. Principal Shadows of the 12 Pentagonal Regular 4-Dimensional Objects (Polytopes). Leonardo 13, 4: 288-294. DOI 10.2307/2688637

Forsyth, Andrew Russell. 1930. Geometry of Four Dimensions. Cambridge: The University Press.

Hanson, Andrew J. and Heng, Pheng Ann. 1991. Visualizing the Fourth Dimension Using Geometry and Light. In: Proceedings of the 2nd conference on Visualization '91: 321-328 DOI 10.1109/ VISUAL.1991.175821

Hinton, Charles Howard. 1880. Speculations on the Fourth Dimension: Selected Writings of Charles H. Hinton. Edited by Rudolf v. B. Rucker, Dover Publications, Inc.

Hinton, Charles Howard. 1904. The Fourth Dimension. London: Swan Sonnenschein \& Co, Ltd., New York: John Lane.

Hoffmann, Christopf M. and Zhou, Jianhua. 1991. Some techniques for visualizing surfaces in fourdimensional space. Computer-Aided Design 23, 1: 83-91. DOI 10.1016/0010-4485(91)90083-9

Kageyama, Akira. (2016.) A Visualization Method of Four Dimensional Polytopes by Oval Display of Parallel Hyperplane Slices. Journal of Visualization 19, 3: 417-422. DOI 10.1007/s12650-0150319-5

Lindgren, Carlos Ernesto S. and Slaby, Steve M.. 1968. Four-Dimensional Descriptive Geometry. New York: McGraw-Hill.

Manning, Henry Parker. 1956. Geometry of Four Dimensions. New York: Dover.

Miyazaki, Koji. 1988. Visualization of a Die and Other Objects in Four-Dimensional Space. Leonardo 21, 1: 56-60. DOI 10.2307/1578417

Monge, Gaspard. 1799. Géométrie descriptive. Paris: Baudouin.

Séquin, Carlo Heinrich. 2002. 3D Visualization Models of the Regular Polytopes in Four and Higher Dimensions. In: Proceedings of Bridges: Mathematical Connections in Art, Music, and Science: 37-48.

Şerbănoiu, Bogdan V., and Şerbănoiu, Adrian A.. 2017. Multidimensional Descriptive Geometry. In: 16th edition National Technical-Scientific Conference, Modern Technologies for the 3rd Millennium, Romania: 257-266.

Stachel, Hellmuth. 1990 The Right-Angle-Theorem in Four Dimensions. Journal for Theoretical Graphics and Computing 3, 1: 4-13.

Volkert, Klaus. 2017. On Models for Visualizing Four-Dimensional Figures. The Mathematical Intelligencer 39, 2: 27-35. DOI 10.1007/s00283-016-9699-1

Weiss, Gunther. 1997. (n,2)-Axonometries and the Contour of Hyperspaces. Journal for Geometry and Graphics 1, 2: 157-167.

Zachariáš, Svätopluk, Velichová, Daniela. 2000. Projection from 4D to 3D. Journal for Geometry and Graphics 4, 1: 55-69.

Zhou, Jianhua. 1991. Visualization of Four Dimensional Space and Its Applications (Ph.D. Thesis). Computer Science Technical Reports, Paper 922. 